6065

February, 1993

$\mathrm{T} / \mathrm{E}$

\title{
Computation of Mini-Jet Inclusive Cross Sections*
}

\author{
Vittorio Del Duca, Michael E. Peskin, and Wai-Keung Tang \\ Stanford Linear Accelerator Center \\ Stanford University, Stanford, California 94309
}

\begin{abstract}
We apply the theory of parton-parton total cross sections at large $s$, due to Lipatov and collaborators, to compute the inclusive cross section for jets which accompany a large $s$ parton scattering process.
\end{abstract}

Submitted to Physics Letters B

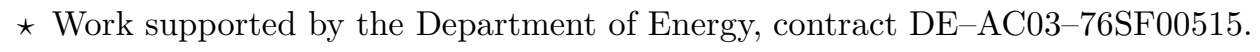


The enormous dynamic range for QCD processes opened by the Tevatron collider has given a new impetus to detailed investigations of the dynamics of quark and gluon scattering. Though the most prominent experimental investigations have involved the cross section for 2 -jet production, there are also new results on 3- and multi-jet processes. In these higher-order processes, there are two or more different Lorentz invariants which set the scale of the momentum transfer, and, typically, these invariants may differ by large factors. The study of QCD processes with large ratios of invariants - semihard processes - is complicated theoretically because it typically involves the resummation of infinite classes of Feynman diagrams. It is complicated experimentally because it requires jet detection in a large rapidity interval. Nevertheless, this regime is a fascinating one, with new and nontrivial applications of QCD perturbation theory. In addition, as Bjorken has recently emphasized, the study of jet correlations over large rapidity intervals may offer interesting signatures of new physics. ${ }^{[1]}$

In this paper, we will discuss the following situation, which involves jet dynamics with a large ratio of invariants. Consider a process in which gluons or quarks scatter with large center-of-mass energy and only moderately large transverse momentum. Call the squared center-of-mass energy of the original parton-parton scattering $s$, and let $m$ be a typical value of the final parton transverse momenta. In the semihard regime, $s>>m^{2}>>\Lambda_{Q C D}^{2}$, Lipatov and collaborators ${ }^{[2-4]}$ have shown that, in this regime, the rapidity interval between the scattered partons is filled in by the radiation of additional gluons, roughly uniformly spaced in rapidity, all with transverse momenta of order $m$. In principle, the properties of these radiated gluons can be observed by choosing events with two jets at large positive and negative rapidity and then measuring the production rate for jets at intermediate rapidities which accompany these jets. In this paper, we will call the jets at large rapidity tagging jets, and we will call the jets that accompany them minijets. A few results on the spectrum of these accompanying gluon jets are known from the work of Balitsky, Fadin, Kuraev, and Lipatov (BFKL), refs. 2-4, and from succeeding work of Levin and Ryskin. ${ }^{[5]}$ In this paper, we return to this question and 
give a systematic procedure for computing the inclusive spectrum of accompanying jets, along with numerical estimates for Tevatron and SSC energies.

\section{Total Cross Section for Tagging Jets}

In order to understand the spectrum of minijets which accompany a set of tagging jets, we should first review the QCD prediction for the total cross section for producing these tagging jets. This prediction has been presented by Mueller and Navelet, ${ }^{[6]}$ using the expression for the asymptotic parton-parton total cross section which is the principal result of the BFKL analysis. We will use the ingredients in this prediction to construct the related inclusive cross sections.

Following Ref. 6, we consider the scattering of two hadrons of momenta $p_{A}$ and $p_{B}$ in the center-of-mass frame, with the $z$ axis along the beam momenta, and we imagine that we tag two jets at the extremes of the Lego plot, with the rapidity interval between them filled with minijets. The tagging jets can be characterized by their transverse momenta and by their longitudinal fractions $x_{A}, x_{B}$ with respect to their parent hadrons. It is simplest to consider the cross section for producing two tagging jets with transverse momenta greater than a minimum value $m$. Then

$$
\begin{aligned}
\frac{d \sigma}{d x_{A} d x_{B}} & \left(A B \rightarrow j\left(x_{A}\right) j\left(x_{B}\right)+X\right) \\
& =\prod_{i=A, B}\left[G\left(x_{i}, m^{2}\right)+4 / 9 \sum_{f}\left[Q_{f}\left(x_{i}, m^{2}\right)+\bar{Q}_{f}\left(x_{i}, m^{2}\right)\right]\right] \cdot \sigma_{t o t}(s)
\end{aligned}
$$

where $s=2 p_{A} \cdot p_{B} x_{A} x_{B}$ is the parton-parton squared center-of-mass energy, and $\sigma_{t o t}$ is the BFKL total cross section for gluon-gluon scattering, which we will discuss in a moment. Eq. (1) includes the effects of quarks using the observation of Combridge and Maxwell that, in a process with large rapidity intervals, the leading contribution to any scattering process comes from gluon exchange in the crossed channel. $^{[7]}$ In writing (1), we assume that the values of $x_{A}, x_{B}$ are sufficiently large that the parton distributions can be computed from ordinary Altarelli-Parisi evolution; semi-hard QCD adds additional complications when these fractions become small. $^{[8]}$ 
The core of eq. (1) is the BFKL function $\sigma_{t o t}(s)$, which is given by

$$
\sigma_{t o t}(s)=\frac{8}{N_{c}^{2}-1} \frac{\pi N_{c}^{2} \alpha_{s}^{2}}{2 m^{2}} F(Y)
$$

where $N_{c}=3$ is the number of colors in QCD, $Y=\log \left(s / m^{2}\right)$, and $F(Y)$ is a dimensionless function which we will discuss below. The strong coupling constant is evaluated at a scale $m^{2}$; the running of $\alpha_{s}$ is subleading in the BFKL theory. Setting $F=1$ gives the cross section for gluon-gluon scattering at the lowest order of QCD, integrated over transverse momenta with $\left|k_{i \perp}\right| \geq m$, in the limit $s>>m^{2}$.

The BFKL theory systematically corrects the lowest-order QCD result for $\sigma_{t o t}$ by summing the leading logarithms of $\left(s / m^{2}\right)$. This is done in three stages, as shown in Fig. 1. First, one simplifies the lowest-order QCD diagrams for multigluon production, shown in Fig. 1(a), for the case in which the emitted gluons are widely separated in rapidity. The gluon emission vertex is replaced by a non-local gaugeinvariant effective vertex ${ }^{[2]}$. Next, one sums the leading corrections to the forward amplitude with color octet in the t-channel, as shown in Fig. 1(b). The result has the form of a Regge pole with an infrared-sensitive trajectory. ${ }^{[3]}$ Finally, one uses this resummed, effective gluon exchange to compute the forward amplitude with color singlet in the $t$-channel. ${ }^{[4]}$ The imaginary part of this object is the gluon-gluon total cross section.

The last two step of this procedure involve the solution of integral equations constructed by BFKL. In the final step, the integral equation can be solved explicitly for the imaginary part of the forward amplitude to find tagging jets with transverse momenta $k_{A \perp}, k_{B \perp}$, both greater than $m$, separated by a rapidity interval $Y$. The solution of the equation involves a Laplace transform with respect to rapidity. Then the amplitude is given by

$$
f\left(k_{A \perp}, k_{B \perp}, Y\right)=\int_{a-i \infty}^{a+i \infty} \frac{d \omega}{2 \pi i} e^{\omega Y} f_{\omega}\left(k_{A \perp}, k_{B \perp}\right),
$$


where the Laplace transform has the representation

$$
f_{\omega}\left(k_{A \perp}, k_{B \perp}\right)=\frac{1}{(2 \pi)^{2}} \sum_{n} e^{i n\left(\phi_{A}-\phi_{B}\right)} \int d \nu \frac{\left(k_{A \perp}^{2}\right)^{-1 / 2+i \nu}\left(k_{B \perp}^{2}\right)^{-1 / 2-i \nu}}{\omega-\omega(n, \nu)} .
$$

In this equation, $\phi_{A}-\phi_{B}$ is the azimuthal angle between the transverse momenta of the tagged jets, and

$$
\omega(n, \nu)=\frac{2 N_{c} \alpha_{s}}{\pi}\left[\psi(1)-\operatorname{Re} \psi\left(\frac{|n|+1}{2}+i \nu\right)\right],
$$

with $\psi(z)$ the standard logarithmic derivative of the Gamma function. Throughout this paper, we ignore azimuthal correlations and keep only the leading, $n=0$, term of (4). Near $\nu=0, \omega(\nu)=\omega(0, \nu)$ has the expansion

$$
\omega(\nu)=A-B \nu^{2}+\cdots
$$

with

$$
A=\frac{4 N_{c} \alpha_{s}}{\pi} \log 2, \quad B=\frac{14 N_{c} \alpha_{s}}{\pi} \zeta(3)
$$

The integral over $\omega$ in (3) can be done easily by picking up the pole. Then we may integrate over the jet transverse momenta to obtain the enhancement factor $F$ in eq. (2). The integrals over $k_{A \perp}$ and $k_{B \perp}$ are singular and depend on the cutoff $m$; this gives the factor $m^{-2}$ in (2). Comparing with that formula more closely, we find

$$
F(Y)=\int_{-\infty}^{\infty} \frac{d \nu}{2 \pi} \frac{1}{\nu^{2}+1 / 4} e^{Y \omega(\nu)}
$$

The exponential growth of $F(Y)$ with the rapidity interval is associated with minijet production. Using (6) to expand about the saddle point at $\nu=0$, we can see that $F(Y)$ has the asymptotic behavior

$$
F(Y) \sim \frac{e^{(4 \log 2) z}}{\sqrt{7 \zeta(3) \pi z / 2}}, \quad \text { with } z=\frac{N_{c} \alpha_{s}}{\pi} Y .
$$

Mueller and Navelet showed that this asymptotic form is an accurate representation for $z>0.2$. 


\section{Mini-jet inclusive cross section}

The BFKL total cross section is a sum over multi-jet emission processes. Thus, it is not difficult to pull this amplitude apart and find the contributions from final states with a jet in a specific region of phase space. The cross section for producing a jet at rapidity $y$ and transverse momentum $q_{\perp}$ in association with the tagging jets described in the previous section may be computed as the sum of diagrams shown in Fig. 2. We use the BFKL propagator (3) to represent the ladder and Lipatov's vertex from ref. 2 to represent the gluon emission. For the moment, we retain the dependence on the transverse momenta $k_{A \perp}, k_{B \perp}$ of the tagging jets. Then the inclusive cross section for minijet emission is given by

$$
\begin{aligned}
\frac{1}{\sigma_{\text {tot }}} \frac{d \sigma}{d y d^{2} q_{\perp} d^{2} k_{A \perp} d^{2} k_{B \perp}}=\frac{16 \alpha_{s} N_{c}}{\pi} \frac{m^{2}}{F(Y)} \int \frac{d^{2} k_{1 \perp}}{(2 \pi)^{2}} \frac{d^{2} k_{2 \perp}}{(2 \pi)^{2}}(2 \pi)^{2} \delta^{(2)}\left(k_{1 \perp}-k_{2 \perp}-q_{\perp}\right) \\
\cdot \frac{1}{k_{A \perp}^{2} k_{B \perp}^{2} q_{\perp}^{2}} f\left(k_{A \perp}, k_{1 \perp}, y_{A}-y\right) f\left(k_{2 \perp}, k_{B \perp}, y-y_{B}\right),
\end{aligned}
$$

where $y_{A}, y_{B}$ are the rapidities of the tagging jets, with $Y=y_{A}-y_{B}$. This formula has been derived previously by Levin and Ryskin.

As before, we ignore all correlations in azimuthal angle by integrating or averaging each transverse momentum over its angle $\phi$. Note that the correlations in $\phi$ are, in any event, subleading. With this simplification, we can represent the momentum delta function in (10) as

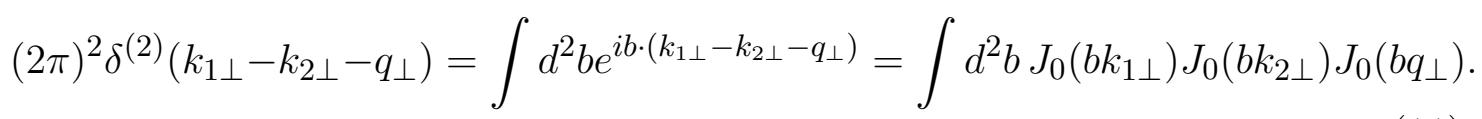

By introducing the representation (4), we can now perform the integrals over the four $k_{i \perp}$. Notice that the integrals over $k_{A \perp}, k_{B \perp}$ depend on the infrared cutoff, as in the derivation of (8). However, the integrals over $k_{1 \perp}, k_{2 \perp}$ converge in the infrared. The resulting expression is a function of two Laplace transform variables, 
which we will call $\nu_{1}, \nu_{2}$. We are left with the integral over $b$, which reduces to

$$
\int_{0}^{\infty} d b b^{-1+2 i\left(\nu_{1}-\nu_{2}\right)} J_{0}\left(b q_{\perp}\right)
$$

To make this integral well-defined, we deform the contours of integration over the $\nu_{i}$ so that $\operatorname{Im}\left(\nu_{2}\right)>\operatorname{Im}\left(\nu_{1}\right)$. We must keep $\left|\operatorname{Im}\left(\nu_{i}\right)\right|<\frac{1}{2}$ in order that the integrals over the $k_{i \perp}$ converge. We will show below that there is a saddle point for the $\nu_{i}$ with this property.

After performing the integral over $b$, we are left with the following expression for the inclusive cross section for the production of one minijet with rapidity $y$ and transverse momentum $q$ in association with tagging jets with transverse momentum greater than $m$ :

$$
\begin{gathered}
\frac{1}{\sigma_{t o t}} \frac{d \sigma}{d y d^{2} q_{\perp}}=\frac{1}{F(Y)} \int_{-\infty}^{\infty} \frac{d \nu_{1}}{2 \pi} \frac{d \nu_{2}}{2 \pi} e^{\omega\left(\nu_{1}\right)\left(y_{A}-y\right)} e^{\omega\left(\nu_{2}\right)\left(y-y_{B}\right)} \frac{N_{c} \alpha_{s}}{\pi^{2} q_{\perp}^{2}}\left(\frac{m^{2}}{q_{\perp}^{2}}\right)^{i\left(\nu_{1}-\nu_{2}\right)}\left(\frac{-i}{\nu_{1}-\nu_{2}}\right) \\
{\left[\frac{1}{\left(\frac{1}{2}-i \nu_{1}\right)} \frac{\Gamma\left(\frac{1}{2}-i \nu_{1}\right)}{\Gamma\left(\frac{1}{2}+i \nu_{1}\right)} \frac{\Gamma\left(1+i\left(\nu_{1}-\nu_{2}\right)\right)}{\Gamma\left(1-i\left(\nu_{1}-\nu_{2}\right)\right)} \frac{\Gamma\left(\frac{1}{2}+i \nu_{2}\right)}{\Gamma\left(\frac{1}{2}-i \nu_{2}\right)} \frac{1}{\left(\frac{1}{2}+i \nu_{2}\right)}\right] .}
\end{gathered}
$$

The variables $\nu_{i}$ are integrated along contours with $\frac{1}{2}>\operatorname{Im}\left(\nu_{2}\right)>\operatorname{Im}\left(\nu_{1}\right)>-\frac{1}{2}$.

The same method yields an integral expression for the $n$-minijet inclusive cross section. The integration variables are the $n+1$ Laplace transform variables of the intermediate BFKL propagators. By the argument given above, these are ordered along the imaginary axis from the tagging jet $B$ to the tagging jet $A$ : $\frac{1}{2}>\operatorname{Im}\left(\nu_{n+1}\right)>\cdots>\operatorname{Im}\left(\nu_{1}\right)>-\frac{1}{2}$. The full inclusive cross section is given by

$$
\begin{aligned}
& \frac{1}{\sigma_{t o t}} \frac{d \sigma}{\prod_{i=1}^{n} d^{2} q_{i \perp} d y_{i}} \\
&= \frac{1}{F(Y)} \prod_{i=1}^{n+1} \int \frac{d \nu_{i}}{2 \pi} e^{\omega\left(\nu_{i}\right)\left(y_{i-1}-y_{i}\right)} \cdot \prod_{i=1}^{n}\left\{\frac{N_{c} \alpha_{s}}{\pi^{2} q_{i \perp}^{2}}\left(\frac{q_{i \perp}^{2}}{m^{2}}\right)^{-i\left(\nu_{i}-\nu_{i+1}\right)}\left(\frac{-i}{\nu_{i}-\nu_{i+1}}\right)\right\} \\
& \cdot\left[\frac{1}{\left(\frac{1}{2}-i \nu_{1}\right)} \frac{\Gamma\left(\frac{1}{2}-i \nu_{1}\right)}{\Gamma\left(\frac{1}{2}+i \nu_{1}\right)}\left\{\prod_{i=1}^{n} \frac{\Gamma\left[1+i\left(\nu_{i}-\nu_{i+1}\right)\right]}{\Gamma\left[1-i\left(\nu_{i}-\nu_{i+1}\right)\right]}\right\} \frac{\Gamma\left(\frac{1}{2}+i \nu_{n+1}\right)}{\Gamma\left(\frac{1}{2}-i \nu_{n+1}\right)} \frac{1}{\left(\frac{1}{2}+i \nu_{n+1}\right)}\right] .
\end{aligned}
$$




\section{Asymptotic Evaluation}

In eq. (9), we simplified the integral formula for the total jet-jet cross section by evaluating the integral asymptotically around an appropriate saddle point. It is straightforward to evaluate the one-jet inclusive cross section asymptotically in the same way. We will find a joint saddle point in the variables $\nu_{1}, \nu_{2}$ and expand the double integral around this point. The expansion will be valid in the limit in which $\left(y_{A}-y\right)$ and $\left(y-y_{B}\right)$ are both large. We neglect the terms in the square brackets in eq. (14), since at asymptotic energies the saddle point will be very close to the origin in the plane $\nu_{1}, \nu_{2}$.

The joint saddle point will occur with both of the $\nu_{i}$ on the imaginary axis: $\nu_{i}=-i n_{i}$. Using the expansion (6), we find the saddle point conditions:

$$
\begin{aligned}
& 2\left(y_{A}-y\right) B n_{1}-\log \left(q_{\perp}^{2} / m^{2}\right)-\frac{1}{n_{1}-n_{2}}=0 \\
& 2\left(y-y_{B}\right) B n_{2}+\log \left(q_{\perp}^{2} / m^{2}\right)+\frac{1}{n_{1}-n_{2}}=0 .
\end{aligned}
$$

From these equations,

$$
n_{1}=\left(y-y_{B}\right) \eta ; \quad n_{2}=-\left(y_{A}-y\right) \eta
$$

with

$$
\eta=\frac{\pi \log \left(q_{\perp}^{2} / m^{2}\right)}{56 N_{c} \zeta(3) \alpha_{s}\left(y_{A}-y\right)\left(y-y_{B}\right)}[1+a]
$$

and

$$
a=\sqrt{1+\frac{112 N_{c} \zeta(3) \alpha_{s}\left(y_{A}-y\right)\left(y-y_{B}\right)}{\pi Y \ln ^{2}\left(q_{\perp}^{2} / m^{2}\right)}} .
$$

Expanding about this saddle point, we find

$$
\frac{1}{\sigma_{t o t}} \frac{d \sigma}{d y d \ln q_{\perp}^{2}}=\frac{N_{c} \alpha_{s}}{2 \pi^{5 / 2}} \sqrt{1-\frac{1}{a}} \exp \left[-\frac{1}{a-1}+1 / 2\right],
$$

with $a$ as in (18). 
According to this equation, the jet inclusive cross section, falls off faster than the scale invariant dependence $\left(d^{2} q_{\perp} / q_{\perp}^{2}\right)$ expected for an approximately fixed value

of $\alpha_{s}$. At small transverse momentum, where we can ignore the $\log \left(q_{\perp}^{2} / m^{2}\right)$ terms in solving (15), there only is a small modification by a factor $\left(q_{\perp}^{2}\right)^{-Y \eta}$. At large transverse momentum, the cross section falls off faster than any power of $q_{\perp}$. This latter, doubly asymptotic, limit has been found previously by Ryskin using another method. $^{[5]}$

\section{Numerical Evaluation}

The formula (13) is also an appropriate starting point for a numerical evaluation of the one jet inclusive cross section. It is important to study the exact behavior of this formula numerically, for two reasons. First, the total rapidity interval for jet-jet scattering processes is not large at energies now available, and so it might be troublesome to divide this interval into several pieces, each of which must be large to justify an asymptotic analysis. Second, this problem is exacerbated by the presence of the poles in (13) which restrict the region of the imaginary axis through which the $\nu_{i}$ contours can pass. At asymptotic energies, the saddle points found in the previous section lie very close to $\nu_{i}=0$, and so these poles are not relevant. However, the evaluation of the saddle point locations with realistic parameters puts $n_{1}$ and $n_{2}$ close to $\pm \frac{1}{2}$. Thus, we might expect large discrepancies for the saddle point result in realistic cases, and, indeed, we will find this below.

To compute the minijet cross section explicitly, we evaluated the double integral in (13) numerically using the contours $\operatorname{Im} \nu_{1}, \operatorname{Im} \nu_{2}= \pm \frac{1}{4}$. We evaluated $\alpha_{s}=$ $\alpha_{s}\left(q_{\perp}\right)$, scaled from $\alpha_{s}\left(m_{Z}\right)=0.12$ using 1-loop evolution with 5 flavors.

For (8), we saw that the saddle point approximation was an accurate one. However, this turns out not to be true for the asymptotic evaluation of (13). In Fig. 3, we compare the exact and asymptotic formulae for the one jet inclusive cross section for gluon-gluon scattering processes at center of mass energies from 1 to $10^{9} \mathrm{TeV}$, with $m=20 \mathrm{GeV}$. There are large discrepancies between the two formulae which disappear only extremely slowly, as the inverse of the logarithm of 
the energy. In fact, the asymptotic formula is never accurate. Since the total cross section (9) rises as a power of $s$, the contribution from the exchange of one gluon ladder must at some point be unitarized by contributions from multiple ladder exchanges. $^{[9]}$ This correction is already important at $100 \mathrm{TeV}$, and changes the one jet inclusive cross section essentially at higher energies.

However, even if the asymptotic formula is not valid, we can estimate the minijet inclusive cross section directly from (13). It is important to recall that this equation, as well as (8), involve additional asymptotic approximations. The BFKL integral equation is derived under the assumption that the emitted gluons are widely separated in rapidity. The solution of the color octet exchange problem shown in Fig. 1(b) involves the assumption that the leading Regge pole in the $\omega$ plane dominates over the remaining singularities. However, there is no reason why either of these effects should lead to large corrections under realistic conditions.

With this set of approximations, then, we present in Fig. 4 predictions from (13) for the minijet inclusive cross section at Tevatron and SSC energies, taking the longitudinal fractions $x_{A}, x_{B}$ of the tagging jets momenta to be 0.1 . The cross section is computed for an observed jet in the center of the rapidity interval between the tagging jets. However, the minijet cross section is almost independent of the position of the jet in the rapidity interval. These curves, and also those of Fig. 3, show a shift of the transverse momentum distribution toward higher values as the energy of the original scattering process - and, therefore, the length of the gluon cascade -increases. This effect can also be seen in eq. (19), although the dependence of that formula on $q_{\perp}$ and $y$ does not match our quantitative results.

The final situation in which we are left is somewhat ambiguous. On one hand, we have improved the understanding of the physics of the BFKL total cross section. We have presented predictions from the BFKL theory for the one minijet inclusive cross section, and we have presented a formula which can be used to evaluate higher order jet correlations. However, we have found that the natural asymptotic evaluations of these formulae are not accurate at the energies of present colliders. 
It will be interesting to see whether the estimates presented in Fig. 4 will be confirmed experimentally. However, we did not succeed in presenting a quantitative and characteristic prediction which can be used to confirm the BFKL resummation. That remains an interesting problem for the future.

\section{Acknowledgements}

We are very grateful to $\mathrm{bj}$. Bjorken and $\mathrm{Al}$ Mueller for encouraging us to investigate this problem. We also thank Lev Lipatov and Eugene Levin for useful discussions. 


\section{REFERENCES}

1. J. D. Bjorken, Phys. Rev. D47 (1992) 101.

2. L. N. Lipatov, Sov. J. Nucl. Phys. 23 (1976) 338.

3. E. A. Kuraev, L. N. Lipatov, and V. S. Fadin, Sov. Phys. JETP 44 (1976) 443, 45 (1977) 199.

4. Ya. Ya. Balitsky and L. N. Lipatov, Sov. J. Nucl. Phys. 28 (1978) 822;

L. N. Lipatov, Sov. Phys. JETP 63 (1986) 904.

5. M. G. Ryskin, Sov. J. Nucl. Phys. 32 (1980) 133;

E. M. Levin and M. G. Ryskin, Sov. J. Nucl. Phys. 32 (1980) 413.

6. A. H. Mueller and H. Navelet, Nucl. Phys. B282 (1987) 727.

7. B. L. Combridge and C. J. Maxwell, Nucl. Phys. B239 (1984) 429.

8. J. C. Collins and R. K. Ellis, Nucl. Phys. B360 (1991) 3;

S. Catani, M. Ciafaloni, and F. Hautmann, Nucl. Phys. B366 (1991) 135.

9. L. V. Gribov, E. M. Levin, and M. G. Ryskin, Phys. Repts. 100 (1983) 1. 


\section{FIGURE CAPTIONS}

1) BFKL resummation: (a) emission of gluons; (b) construction of the octet amplitude; (c) construction of the singlet amplitude.

2) Sum of graphs leading to the inclusive jet cross section.

3) Comparison of the exact and asymptotic evaluations of the one jet inclusive cross section as a function of the jet transverse momentum, at the parton center-of-mass energies $\sqrt{s}=1,10^{3}, 10^{6}, 10^{9} \mathrm{TeV}$, and $m=20 \mathrm{GeV}$. The upper curves represent the exact evaluation of integral (13), and the lower curves its asymptotic evaluation (19).

4) One jet inclusive cross section as a function of the transverse momentum of the jet for typical Tevatron and SSC energies. We choose $\sqrt{s}=0.18$ and $4 \mathrm{TeV}$ respectively for the Tevatron and the SSC parton center-of-mass energies, and we label the curves according to the minimum jet transverse momentum. 